\title{
An unusual cause of bowel perforation associated with necrotising soft tissue infection of the upper limb
}

Case letter

Rachit Datta, BMedSci

Medical Student, Bond University, Gold Coast, QLD, Australia

rachit.datta@student.bond.edu.au

Andrew McBride, Bphty, MBBS

Orthopaedic SET Trainee

Department of orthopaedics, Gold Coast University Hospital, QLD, Australia;

Ruth-Ellen Grace Marks, BMed, DCH, FRACGP, GradCertHIthProfEd

Advanced Trainee in Emergency Medicine, Department of Emergency Medicine, Gold Coast University Hospital, QLD, Australia.

Gerben Keijzers, MSc (Biomed Health Sci), MB BS, FACEM, PhD

Emergency Physician, Department of Emergency Medicine, Gold Coast University Hospital, QLD, Australia; Professor (adjunct), Bond University, Gold Coast, QLD, Australia; Griffith University, Gold Coast, QLD, Australia

Gerben.Keijzers@health.qld.gov.au 


\section{Dear Editor}

A 58 year-old male presented to the emergency department (ED) with a twelve hour history of atraumatic right elbow pain, fevers and increasing lethargy. The patient also reported abdominal pain at triage, which commenced over 24 hours before presentation as well as malaise, rigours and diaphoresis. The abdominal pain had fully subsided by the time he was assessed by the treating doctor at 0150AM. He had no past medical history and did not take regular medication.

The vitals on arrival were; temperature $38.9^{\circ} \mathrm{C}$, heart rate $91 / \mathrm{min}$, respiratory rate $20 / \mathrm{min}$, blood pressure $115 / 67 \mathrm{~mm} \mathrm{Hg}$ and pulse oximetry $99 \%$ on room air. His abdomen was soft and non-tender on examination with no signs of peritonism. Within one hour of being initially seen by the treating clinician his right elbow had a rapidly evolving purpuric rash surrounding the olecranon. Crepitus was absent on palpation. There was severe limitation in movement of the elbow, wrist and shoulder due to substantial pain. The pain was deemed out of proportion and he required multiple doses of intravenous opioids. Sensation remained intact across all dermatomes with a strong and regular pulse and a capillary refill less than two seconds. A plain radiograph of the right upper limb showed subcutaneous and deep layer gas (Fig 1a).

As a necrotising soft tissue infection (NSTI) was suspected, broad spectrum intravenous antibiotics (Flucloxacillin 2g, Meropenem 1g, Vancomycin $2 \mathrm{~g}$ and Lincomycin 600mg) were initiated. After orthopaedic surgical consultation the patient was immediately booked for surgical debridement. At this stage the patient's vitals were RR 16/min, pulse 90 bpm, BP 141/68mmHg, pulse oximetry $100 \%$ on room air. Pathology results included a WBC of 19.1, CRP of 174 and venous gas with a PH: 7.43 , bicarbonate of $23 \mathrm{mmol} / \mathrm{L}$ and lactate of $4.2 \mathrm{mmol} / \mathrm{L}$. While awaiting the on-call theatre staff, a CT scan of the upper limb was performed to delineate the extent of the NSTI without delaying the patient's need for emergent surgical care. The CT scan showed extensive gas in the subcutaneous and fascial layers from the midforeram to the proximal humerus extending to the infraclavicular region and into the axilla (see Fig 1b). The arm was placed on top of the abdomen during the scanning procedure, and on review of the limb images an incidental finding of a localised caecal perforation was noted by the reporting radiology registrar (Fig $1 \mathrm{~b}$ ). 
Emergency surgical debridement in the operating theatre commenced at 5:30am (3.5 hrs after initial treating ED doctor assessment). Upper limb fasciotomy revealed classic "dishwasher" fluid indicating non-viable necrotic tissue. The patient deteriorated during procedure and source control consisted of emergency proximal humeral amputation at the level of the surgical neck. In addition, the patient underwent a right open hemicolectomy with end ileostomy and stoma formation. Histological examination of the surgical specimen showed Large B-cell NonHodgkin's lymphoma of the caecum with transmural necrosis and macroscopic perforation. Tissue and fluid culture from the right forearm revealed Clostridium Septicum (C. septicum). C. septicum is a highly virulent pathogen associated with colorectal malignancy and haematological malignancy. ${ }^{1} \mathrm{NSTI}$ or clostridial myonecrosis has been implicated in approximately $60-80 \%$ of C. septicum cases. Sepsis from C. septicum has a mortality rate of $60 \%$ and reported rates of underlying malignancy as a result of $C$. septicum infection range from 50 to $85 \%{ }^{1}$

The association between $C$. Septicum and malignancy is of significance as it is often indolent without external signs of infection. The environment of a tumour is advantageous for germination of spores and evolving infection. Inflammation in the bowel can lead to translocation of localised infection producing spontaneous or remote myonecrosis in the absence of prior trauma. ${ }^{1} \mathrm{NSTI}$ are destructive, lifethreatening infections affecting soft tissue that can move along facial planes. Prompt diagnosis of NSTI is vital to minimise morbidity (such as amputation) and mortality. ${ }^{2}$

Delay in diagnosis of NSTI occurs in $46-80 \%$ of patients. ${ }^{3}$ Early in its course, signs such as swelling, pain and erythema can be indistinguishable from cellulitis or abscess formation. Clinicians are faced with the challenging task of identifying the rare NSTI from the very common soft tissue infection without deep space necrosis. In cases where non-specific symptoms predominate, the 'laboratory risk indicator for necrotizing fasciitis score' (LRINEC) has been created to discriminate between NSTI and non-necrotizing soft-tissue infection. ${ }^{4}$ Using variables widely available this can be an additional tool for clinicians to assess patients where the clinical picture may not be as evident as our patient. Intermediate and high-risk LRINEC scores (score $>6$ ) have a PPV of $92 \%$ and a NPV of $96 \%,{ }^{5}$ with our patient having a score 
of 7 (CRP>150: 4 points; WBC 19.1: 1 point; Hgb 120: 1 point; Sodium 136mmol/L: 0 points; Creatine 87 umol/L: 0 points; Glucose $11 \mathrm{mmol} / \mathrm{L}: 1$ point).

The patient was discharged home 28 days after presentation and was independent in daily activities, and planned to commence chemotherapy for his NHL.

\section{References}

1) Jessamy K, Ojevwe FO, Ubagharaji E, Sharma A, Anozie O, Gilman CA, Rawlins S. Clostridium septicum: an unusual link to a lower gastrointestinal bleed. Case reports in gastroenterology. 2016;10(2):489-93.

2) Cheng NC, Su YM, Kuo YS, Tai HC, Tang YB. Factors affecting the mortality of necrotizing fasciitis involving the upper extremities. Surgery today. 2008 Dec 1;38(12):1108-13.

3) Khan AA, Davenport K: A reminder of the association between Clostridium septicum and colonic adenocarcinoma. Int Semin Surg Oncol 2006;3: 12.

4) Goldstein EJ, Anaya DA, Dellinger EP. Necrotizing soft-tissue infection: diagnosis and management. Clinical Infectious Diseases. 2007 Mar 1;44(5):705-10.

5) Wong CH, Khin LW, Heng KS, Tan KC, Low CO. The LRINEC (Laboratory Risk Indicator for Necrotizing Fasciitis) score: a tool for distinguishing necrotizing fasciitis from other soft tissue infections. Critical care medicine. 2004 Jul 1;32(7):1535-41. 
Figure 1a

Legend

Plain radiograph showing superficial and deep layer gas formation

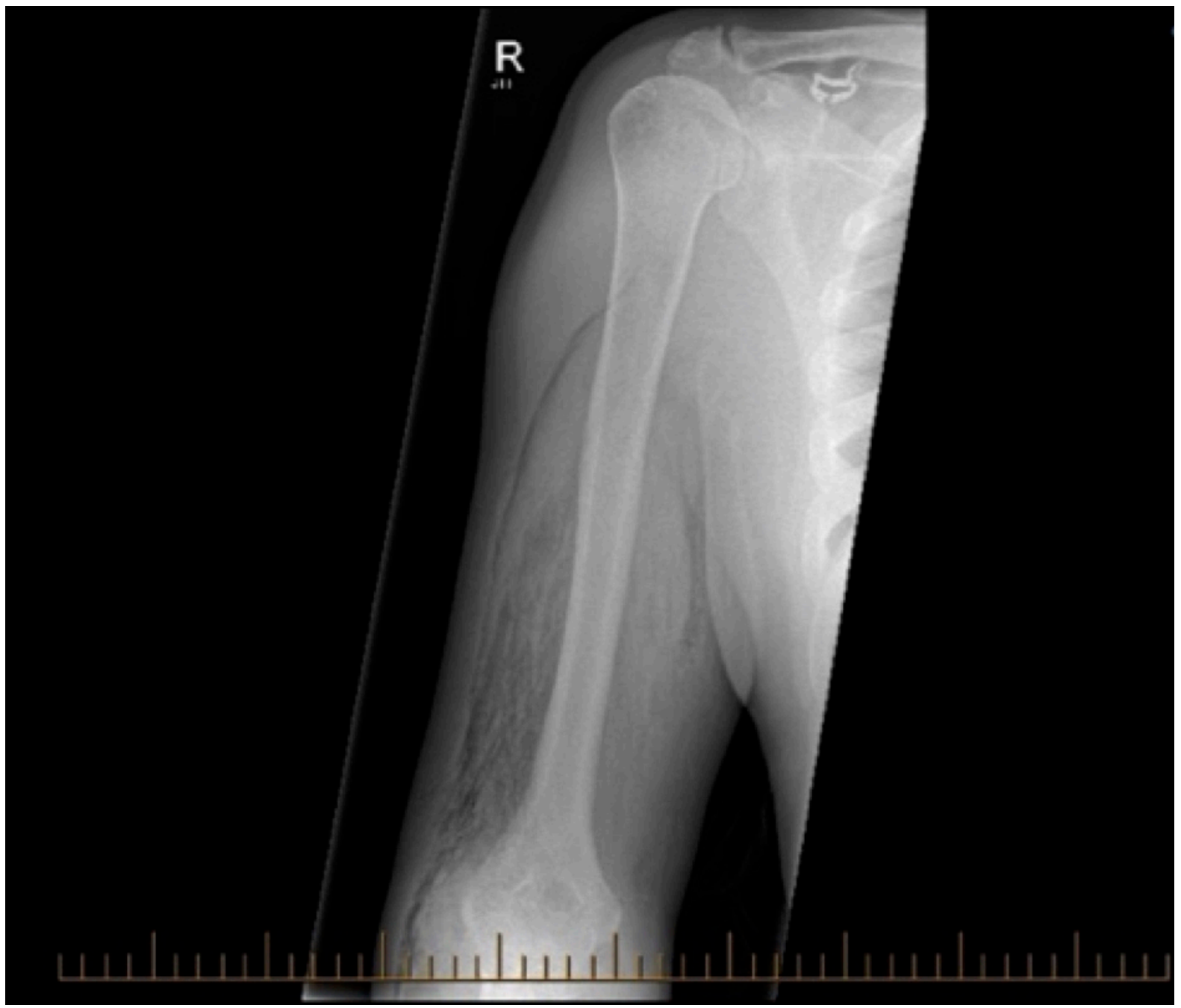


Figure 1b

Legend

Computed tomogram showing limb and abdomen

Large yellow arrow: Caecal perforation

Small yellow arrow: Gas in bowel

Small blue arrow: Gas in tissue planes

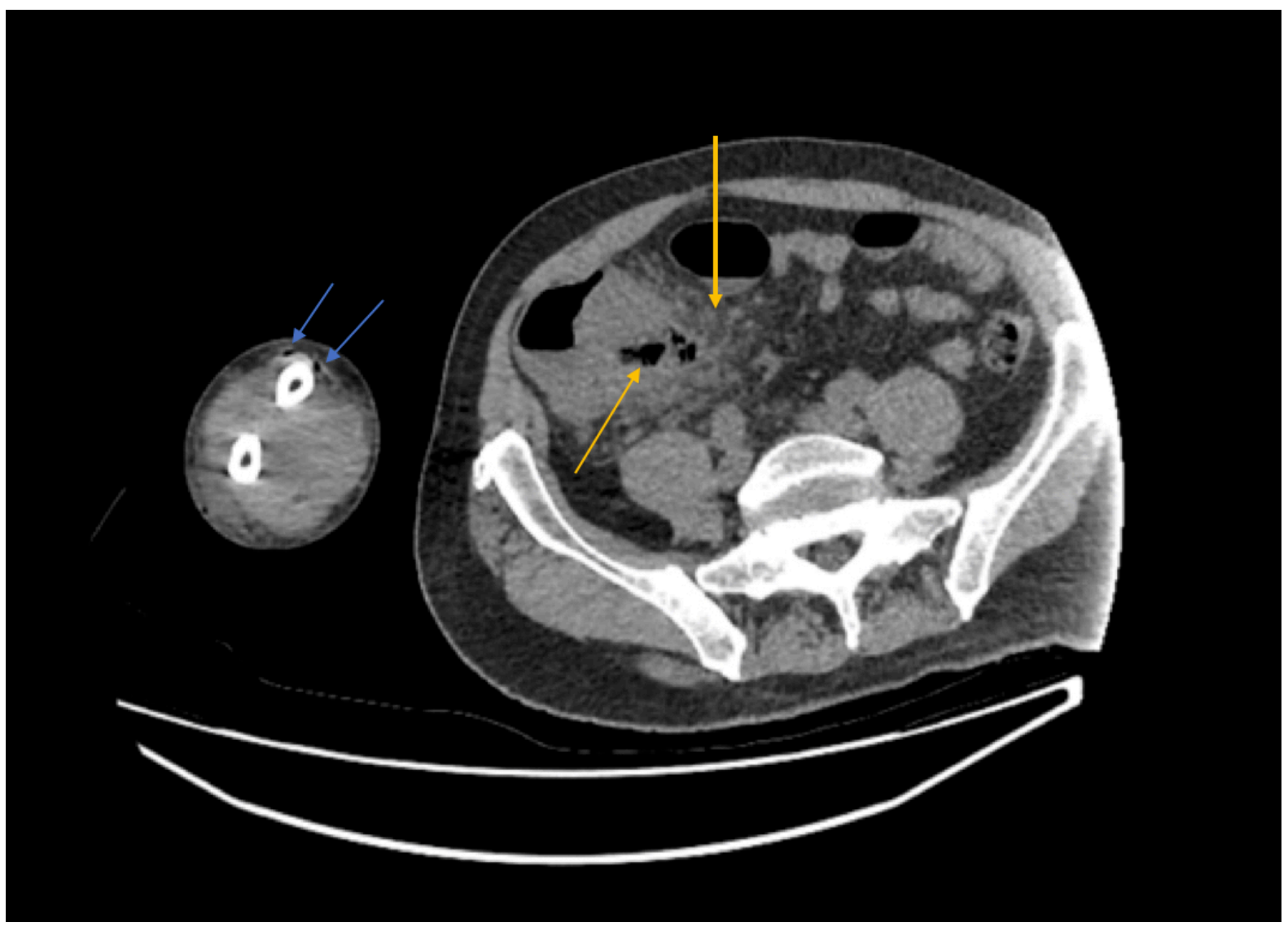

